\title{
THE ABSOLUTE MINIMUM OF A DEFINITE INTEGRAL IN A SPECIAL FIELD*
}

BY

\section{E. J. MILES}

There are many examples illustrating the general theory of relative minima in the calculus of variations, but comparatively few cases have been given where questions of absolute minima are discussed. This is due no doubt to the fact that the conditions which must be set down in order to establish a priori the existence of an absolute minimum are very strong. If the existence of an absolute minimum has been established, the minimizing curve is contained among those furnishing relative minima.

In this paper it is intended to discuss the absolute minimum of a definite integral

$$
J=\int_{t_{0}}^{t_{1}} F\left(x, y, x^{\prime}, y^{\prime}\right) d t,
$$

in a special field. In the first section the properties of the function $F$ and of the admissible curves through the fixed end-points 0 and 1 are given, the properties ordinarily imposed in the Weierstrass theory. Then, under the assumptions made, it is known that there exists a one-parameter family of extremals passing through the point 0 . This family will in general have an envelope and it is customary to assume that the enveloping curve has no singularities in the region under discussion. In $\S 2$, on the contrary, some properties of a one-parameter family of extremals whose envelope has a cusp will be discussed. Such families have been considered by KNESER $\dagger$, OsGOOD $\ddagger$, HAHN $\S$, LINDEBERG $\|$, who have been concerned for the most part with the minimizing properties of the extremal passing through the cusp point. In $\S 2$ a discussion is given of the way in which the extremals cover the region near the cusp point. It is shown

* Presented to the Society, December 29, 1910.

† Mathematische Annalen, vol. 50 (1898), p. 27; and Lehrbuch der Variationsrechnung, sections $24,25$.

†Transactions of the American Mathematical Society, vol. 2 (1901), p. 166.

Sitzungsberichte der Kaiserlichen Akademie der Wissenschaften in Wien, vol. 118 (1909), Abtheilung IIa, p. 99. The conditions under which HABN works are weaker than those of OsGood.

U Mathematische Annalen, vol. 59 (1904), p. 321 . He considers the curves in the form $y=y(x)$. 
that outside the $\mathrm{V}$-shaped cusp region shown in Fig. 1 there is a single extremal through each point, while for points within there are three, of which one has already touched the envelope.

In $\S 3$ the field about an extremal arc whose end points are conjugate is discussed, under the assumption that the conjugate point is a cusp point in the envelope. It is found that the field can be regarded as a three-sheeted surface similar to a Riemann surface, the sheets being joined along the two branches of the envelope. Then in $\S 4$ it is shown that the envelope law for the integral holds along either branch of the envelope. In $\$ 5$ a curve $M$ in the V-shaped cusp region is determined which marks the cessation of absolute minimum in the field. This curve is continuous, passes through the cusp point 1 and is such that each extremal ceases to furnish an absolute minimum as soon as it meets this curve. Finally in $\S 6$ sufficient conditions for an absolute minimum in the field are considered.

\section{§ 1. Properties of the function $F$.}

The conditions for the existence of an absolute minimum * in a region $R$, for the case of the definite integral

$$
J=\int_{t_{0}}^{t_{1}} F\left(x, y, x^{\prime}, y^{\prime}\right) d t
$$

with fixed end points, were first given by Hilbert. $\dagger$

In the present paper it is proposed to study the question of the absolute minimum in a special field. Consider the extremal arc $C_{01}$,

$$
x=\varphi(t), \quad y=\psi(t), \quad t_{0} \leqq t \leqq t_{1}
$$

of class $C^{\prime} \ddagger$, without singular points, and joining two fixed points 0 and 1 . In a neighborhood of $C_{01}$ suppose that $F\left(x, y, x^{\prime}, y^{\prime}\right)$ is of class $C^{\text {IV }}$ and satisfies the homogeneity condition

$$
F\left(x, y, l i x^{\prime}, k y^{\prime}\right)=k F\left(x, y, x^{\prime}, y^{\prime}\right)
$$

for all values of $x^{\prime}, y^{\prime}$ for which

$$
x^{2}+y^{\prime 2} \neq 0
$$

* For a discussion of the absolute extremum, see Bolza, Vorlesungen über Variationsrechnung, Chapter IX.

† Jahresbericht der Deutschen Mathematiker-Vereinigung, vol. 8 (1899), p. 184.

$\ddagger$ A function is of class $C^{(n)}$ if it possesses continuous derivatives up to and including those of the $n$th order. 
Along $C_{01}$ it will be supposed also that $F$ and the function

are positive.

$$
F_{1}=\frac{F_{x^{\prime} x^{\prime}}}{y^{\prime 2}}=-\frac{F_{x^{\prime} y^{\prime}}}{x^{\prime} y^{\prime}}=\frac{F_{y^{\prime} y^{\prime}}}{x^{\prime 2}}
$$

Under these assumptions it can be shown* that through any point on the extremal $C_{01}$, there passes a one-parameter family of extremals

$$
x=\varphi(t, a), y=\psi(t, a)
$$

which has the properties:

(1) the extremal $C_{01}$ is given by $a=a_{1}$;

(2) the functions $\varphi, \varphi_{t}, \varphi_{t t}, \psi, \psi_{t}, \psi_{t t}$ are of class $C^{\prime \prime}$ in a certain vicinity of the values

$$
t_{0} \leqq t \leqq t_{1}, \quad a=a_{1}
$$

(3) for every $(t, a)$ in this vicinity the extremal $C_{a}$ lies entirely in the region in which the function $F$ has the continuity properties described above, and is such that

$$
\begin{gathered}
F_{1}\left[\varphi(t, a), \psi(t, a), \varphi_{t}(t, a), \psi_{t}(t, a)\right]>0, \\
\varphi_{t}^{2}(t, a)+\psi_{t}^{2}(t, a)>0 ;
\end{gathered}
$$

(4) the functional determinant

$$
\Delta(t, a)=\frac{\partial(\varphi, \psi)}{\partial(t, a)}
$$

is such that for $a=a_{1}$ it does not vanish identically as a function of $t$.

§ 2. Properties of a one-parameter family of extremals whose envelope has a cusp.

It is desired to consider the one-parameter family of extremals through the fixed point 0 given by the equation $\left(C_{a}\right)$. Suppose the following hypotheses concerning the extremals and their envelope are inade:

$$
\begin{aligned}
\text { I. } & \varphi_{t}\left(t_{1}, a_{1}\right)>0 ; \\
\text { II. } & \Delta\left(t_{1}, a_{1}\right)=0, \Delta_{t}\left(t_{1}, a_{1}\right)<0 ; \\
\text { III. } & \varphi_{t}\left(t_{1}, a_{1}\right) \Delta_{a}\left(t_{1}, a_{1}\right)-\phi_{a}\left(t_{1}, a_{1}\right) \Delta_{t}\left(t_{1}, a_{1}\right)=0 \text {, } \\
& \psi_{t}\left(t_{1}, a_{1}\right) \Delta_{a}\left(t_{1}, a_{1}\right)-\psi_{a}\left(t_{1}, a_{1}\right) \Delta_{t}\left(t_{1}, a_{1}\right)=0 ;
\end{aligned}
$$

IV. for $a<a_{1}, \varphi_{t}[t(a), a] \Delta_{a}[t(a), a]-\varphi_{a}[t(a), a] \Delta_{t}[t(a), a]<0$, for $a>a_{1}, \varphi_{t}[t(a), a] \Delta_{a}[t(a), a]-\varphi_{a}[t(a), a] \Delta_{t}[t(a), a]>0$;

where $t=t(a)$ is the function defining the envelope of the family $\left(C_{a}\right)$, as will be explained presently.

* Bouza, loc. cit., \$27d. See also Buss, The solutions of differential equations of the first order as functions of their initial values, Annals of Mathematics, vol. 6 (1905). 
It is seen at once that no generality is lost in making the first hypot!.esi-, since $C_{01}$ has not a singular point at 1 . It could be effected by choosing a proper coördinate system.

The second one implies that the extremal $C_{01}$, which may also be denoted by $C_{1}$, has a point of contact with the envelope of the family $\left(C_{a}\right)$ at 1 .

Now consider the equation $\Delta(t, a)=0$ defining points on the envelope. Since $\Delta$ is a solution of the Jacobi equation, it follows that $\Delta_{t}$ can never vanish simultaneously with $\Delta$, and therefore that $\Delta_{t}\left(t_{1}, a_{1}\right) \neq 0 .^{*}$ If it is not negative change the parameter $a$ into $-a$. Hence in the vicinity of the point $\left(t_{1}, a_{1}\right)$ the equation

$$
\Delta(t, a)=0
$$

can be solved for $t$ in terms of $a$, the result being a function of the form

$$
\left(E_{t a}\right) \quad t=t(a), \quad a_{1}-e_{1} \leqq a \leqq a_{1}+e_{1},
$$

which reduces to $t_{1}$ for $a=a_{1}$, and is of class $C^{\prime}$. When expressed in terms of the parameter $a$, the equation of the envelope $E$ in the $x y$-plane then becomes

$$
\left(E_{x y}\right) \quad x=\varphi[t(a), a]=\bar{x}(a), \quad y=\psi[t(a), a]=\bar{y}(a) .
$$

The third and fourth hypotheses determine the nature of this envelope in the neighborhood of the point 1 . By differentiating, the derivatives determining the direction of the tangent are seen to be

$$
\begin{aligned}
& \bar{x}_{a}=\frac{-\varphi_{t}[t(a), a] \Delta_{a}[t(a), a]+\varphi_{a}[t(a), a] \Delta_{t}[t(a), a]}{\Delta_{t}[t(a), a]}, \\
& \bar{y}_{a}=-\frac{-\psi_{t}[t(a), a] \Delta_{a}[t(a), a]+\psi_{a}[t(a), a] \Delta_{t}[t(a), a]}{\Delta_{t}[t(a), a]} .
\end{aligned}
$$

From the third hypothesis it follows that the numerators of these two derivatives are zero at the value $a_{1}$ for which $t\left(a_{1}\right)=t_{1}$. Consequently the envelope $E_{x y}$ has here a singular point. But from the fourth hypothesis it follows that $\bar{x}(a)$ has a minimum at the point 1 , since the derivative $\bar{x}_{a}(a)$ is negative for $a<a_{1}$ and positive for $a>a_{1}$; that is, the two branches of $E$, corresponding to $a\left\langle a_{1}\right.$ and $a>a_{1}$, both lie to the right of the ordinate $x=x_{1}$, as in Fig. 1 .

The curve $E_{x y}$ is tangent to the extremal $C_{a}$ when $a \neq a_{1}$, since along it

and therefore

$$
\Delta=0, \quad \varphi_{a}=k \varphi_{t}, \quad \psi_{a}=k \psi_{t},
$$

$$
\bar{x}_{a}=\varphi_{t} t_{a}+\varphi_{a}=\left(t_{a}+k\right) \varphi_{t}, \quad \bar{y}_{a}=\psi_{t} t_{a}+\psi_{a}=\left(t_{a}+k\right) \psi_{t} .
$$

The two branches for $a<a_{1}$, and $a>a_{1}$ are both tangent to $C_{1}$ at 1 , since for $a \neq a_{1}$ the tangents to $C_{a}$ and $E_{x y}$ coincide, and the tangent to $C_{a}$ approaches

\footnotetext{
* See Bolza, loc. cit., § $29 b$.
} 
that of $C_{1}$ as $(t, a)$ approaches the value $\left(t_{1}, a_{1}\right)$. But since by hypotheses II and IV the derivative $\bar{x}_{a}(a)$ is positive for $a>a_{1}$ and negative for $a<a_{1}$, it follows that the direction of the positive tangent to the branch $a>a_{1}$ coincides with that of $C_{1}$ at 1 , while the direction of the tangent to the branch $a<a_{1}$ is the opposite to that of $C_{1}$. Hence the envelope has a cusp at 1 similar to that shown in Fig. 1.

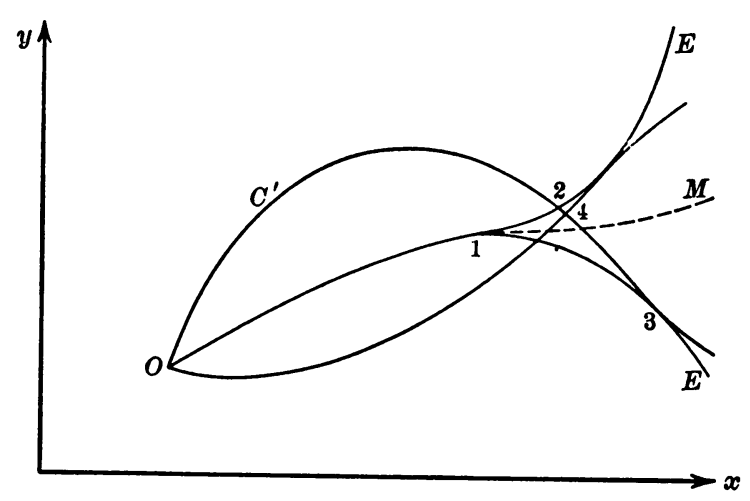

Fig. 1.

With these hypotheses in mind it is proposed to study the solutions of equations $\left(C_{a}\right.$ ) for $t$ and $a$ as functions of $x$ and $y$ in the neighborhood of the point 1 . It can be shown that a region

$$
\left|t-t_{1}\right| \leqq d, \quad\left|a-a_{1}\right| \leqq e
$$

and a constant $\delta$ can be selected in such a way that the following conditions are satisfied:

1) In $R_{t a}$ both $\varphi_{t}$ and $\Delta_{t}$ remain different from zero;

2) every ordinate in the interval $\left|x-x_{1}\right| \leqq \delta$ is crossed once and but once by each extremal arc defined by $R_{t a}$;

3) for ordinates $x_{1}-\delta \leqq x<x_{1}$ there is no value of $a$ for which the envelope $E_{x y}$ intersects the ordinate;

4) each ordinate satisfying $x_{1}<x \leqq x_{1}+\delta$ is cut once and but once by each branch of $E_{x y}$;

5) on the interval $x_{1}-\delta \leqq x \leqq x_{1}+\delta$ the extremals corresponding to $a_{1}+e$ and $a_{1}-e$ have no points in common with $C_{01}$ or the envelope $E_{x y}$.

The first condition is evidently possible since $\varphi_{t}$ and $\Delta_{t}$ are continuous and different from zero at $\left(t_{1}, a_{1}\right)$. From this it also follows at once that the curve $E_{t a}$ defined above divides the region $R_{t a}$ into right and left portions denoted by $R_{t a}^{\prime \prime}$ and $R_{t a}^{\prime}$ such that for all points in $R_{t a}^{\prime}$ the function $\Delta(t, a)$ is positive, while for all points in $R_{t a}^{\prime \prime}$ it is negative. Since the extremal $C_{01}$ crosses the 
ordinate $x=x_{1}$, the second may be fulfilled by choosing a $\delta$ first and then restricting $e$. To satisfy the third and fourth, restrict $e$ if necessary so that the

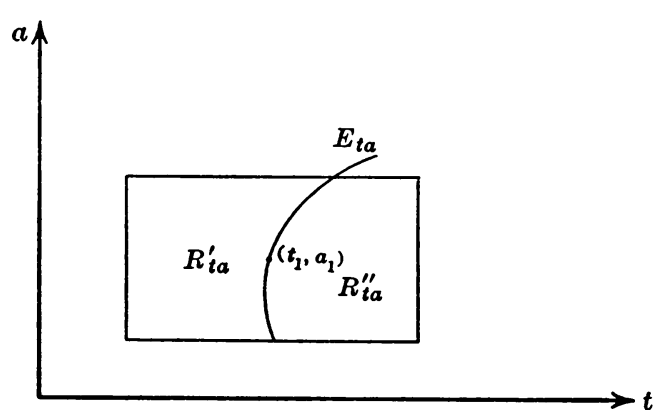

Fig. 2. hypothesis IV holds on the whole of the intervals $a_{1}-e \leqq a<a_{1}$ and $a_{1}<a \leqq a_{1}+e$, and select $\delta$ so small that each branch of the envelope $E_{x y}$ certainly cuts the ordinates of the interval $x_{1}<x \leqq x_{1}+\delta$.

Consider now the unique value of $t$ for which a given extremal corresponding to a value $a$ crosses the ordinate $x$. This is a function $t(x, a)$ which satisfies the equation

$$
x=\varphi(t, a),
$$

and by the usual theorems on implicit functions is therefore of class $C^{\prime}$ for the region

$$
\left|x-x_{1}\right| \leqq \delta, \quad\left|a-a_{1}\right| \leqq e .
$$

The only points $(x, y)$ in the interval $\left|x-x_{1}\right| \leqq \delta$ corresponding to the region $R_{t a}$ by means of the equations

$$
x=\varphi(t, a), \quad y=\psi(t, a)
$$

are now those which satisfy the equation

$$
y=\psi[t(x, a), a]=\gamma(x, a) .
$$

For, if $(x, y, t, a)$ are a set of solutions of $\left(C_{a}\right)$, the value of $t$ would have to be $t(x, a)$, and equation (3) would therefore necessarily be satisfied.

The derivative of $\gamma(x, a)$ for $a$ is readily found to be

$$
\begin{aligned}
\gamma_{a}(x, a) & =\frac{-\psi_{t}[t(x, a), a] \varphi_{a}[t(x, a), a]+\psi_{a}[t(x, a), a] \varphi_{t}[t(x, a), a]}{\varphi_{t}[t(x, a), a]} \\
& =\frac{\Delta[t(x, a), a]}{\varphi_{t}[t(x, a), a]} .
\end{aligned}
$$

Hence on the ordinates $x_{1}-\delta \leqq x \leqq x_{1}$ the value of $y$ constantly increases as $a$ varies from $a_{1}-e$ to $a_{1}+e$. At $x=x_{1}$ the extremals $C_{a_{1}+e}, C_{a_{1}-e}$ are distinct from $C_{01}$ and $E_{x y}$. Hence the value of $\delta$ can be so chosen that condition 5 ) is satisfied. On any ordinate $x_{1}<x \leqq x_{1}+\delta$ the value of $y$ first increases from a point on $C_{a_{1}-e}$ until a point of the upper branch $a<a_{1}$ of the envelope is reached, when $\Delta$ changes sign. Then $y$ decreases to a point on the lower branch, and lastly increases again to a point on $C_{\omega_{1}+e}$. 
It is clear then that to any point $(x, y)$ in the $\mathrm{V}$-shaped region between the branches of the envelope in Fig. 1, there correspond by means of equations $\left(C_{a}\right)$ three and but three values of $(t, a)$ in the region $R_{t a}$, while for points outside of the $V$ there is but one. The values of the functions $t(x, y), a(x, y)$ so defined are associated into branches which are of class $C^{\prime}$ for points not on the envelope, since for such values $(t, a)$ the functional determinant of equations $\left(C_{a}\right)$ is different from zero. Along the envelope one branch retains its continuity and differentiability, and it is not difficult to show that at a point $\left(x^{\prime}, y^{\prime}\right)$ on the envelope the other two are continuous and approach the same limiting value when $(x, y)$ approaches $\left(x^{\prime}, y^{\prime}\right)$ from the interior of the $V$-shaped region, although they are not necessarily differentiable.

Suppose then a surface similar to a Riemann surface covering the region $R_{x y}$ as shown in Fig. 3 .

To each point $(x, y)$ of this surface there corresponds one and but one solution $[t(x, y), a(x, y)]$ of the equations $\left(C_{a}\right)$ in the region $R_{t a}$. To each point of the V-shaped leaf $L_{x y}^{\prime \prime}$ corresponds one and but one

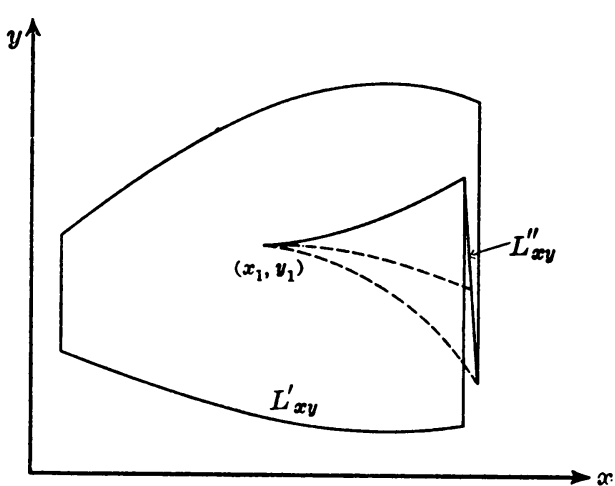

Fig. 3. point of the region $R_{t a}^{\prime \prime}$ to the right of the arc $E_{t a}$ in Fig. 2, while to each point of the second leaf $L_{x y}^{\prime}$ of the Riemann sheet there corresponds one and but one point in $R_{t a}^{\prime}$ on the left of $E_{t a}$.

Interpreted in terms of the extremals it can then be stated that through any

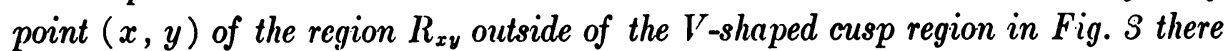
passes one and but one extremal belonging to the family $\left(C_{a}\right)$. Through any point $(x, y)$ of the $V$-shaped cusp region in $R_{x y}$ there pass three extremals. The functions $t(x, y), a(x, y)$ so defined are associated into branches corresponding to the sheets $L_{x y}^{\prime}$ and $L_{x y}^{\prime \prime}$, and are of class $C^{\prime}$ except at points of the envelope, where they are, however, continuous.

\section{\$2. The field about the extremal $C_{01}$.}

Consider the arc $C_{01}$ and the envelope $E$. A certain neighborhood ( $\rho$ ) of $C_{01}$ can be selected as shown in Fig. 4 in such a way that through each point of the neighborhood with the exception of 0 and the $V$-shaped cusp region bounded by the envelope there passes one and but one extremal, while, as was seen above, through each point of the $\mathrm{V}$-shaped region there pass three of the extremals $\left(C_{a}\right)$.

For, as has been shown, ${ }^{*}$ a circle of radius $\left(\rho^{\prime}\right)$ can be found about the point

* Bolza, loc. cit., \& $33 a$. 
0 which is simply covered by the extremals $\left(C_{a}\right)$, whose boundary contains at least one point 4 of the arc $C_{01}$. Furthermore the proof of the preceding section shows the existence of a region $R_{x y}$ about the point 1 which is multiply covered as has been stated in $\S 1$, and whose boundary intersects $C_{01}$ in a second point 5. The usual proof for the existence of a field about an arc not containing a pair of conjugate points shows that a neighborhood $\left(\rho^{\prime \prime}\right) *$ of the $\operatorname{arc} C_{45}$ can be found which is simply covered, and a neighborhood ( $\rho$ ) (Fig. 4) can thus be selected every point of which lies within the region defined above.

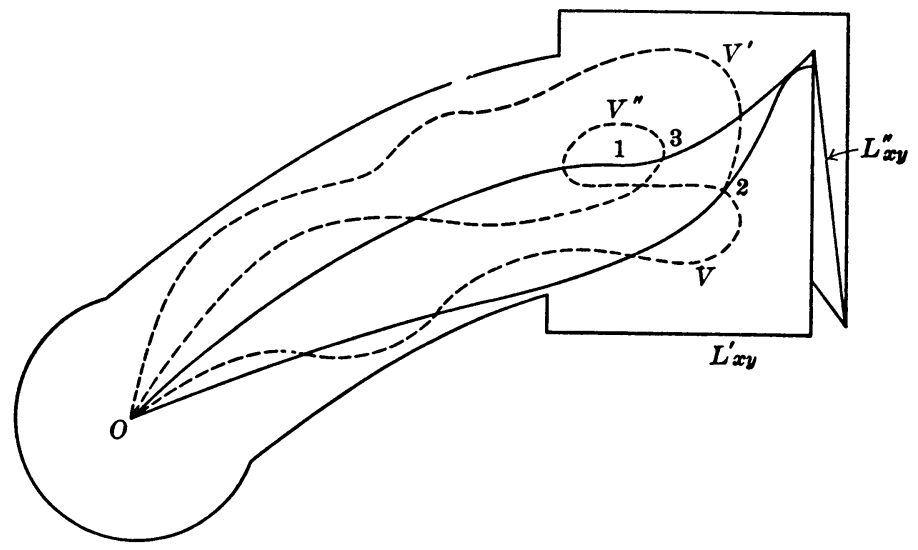

FIG. 4.

The field can be thought of as a Riemann surface on which the functions $t(x, y), a(x, y)$ defining the extremal through the point $(x, y)$ of the surface are of class $C^{\prime}$, with the exception of the point 0 and the cuspidal edges of the surface.

\section{§3. The envelope law.}

The proof of the so-called envelope law in the case at hand when the envelope has a cusp, can be readily made in the ordinary way when the branches of the envelope are considered separately. For either branch the sign of the parameter $a$ can always be so chosen that along the branch of the envelope under consideration the positive tangents of the envelope and extremal coincide.

Suppose then one considers the set of extremals $\left(C_{a}\right)$ passing through 0 for the fixed parameter value $t=t_{0}$ and touching the lower branch of the envelope. It will be shown that the value of the integral $J$,

$$
\begin{aligned}
J\left(a_{2}\right)= & J\left(C_{02}\right)+J\left(E_{23}\right) \\
= & \int_{t_{0}}^{t_{3}} F\left[\varphi\left(t, a_{2}\right), \psi\left(t, a_{2}\right), \varphi_{t}\left(t, a_{2}\right), \psi_{t}\left(t, a_{2}\right)\right] d t \\
& \quad+\int_{a_{2}}^{a_{3}} F\left(\bar{x}, \bar{y}, \bar{x}_{a}, \bar{y}_{a}\right) d a,
\end{aligned}
$$

* Bolza, loc. cit., \& $31 a$. 
taken along the extremal $C_{02}$ and then along the envelope $E$ from the point 2 to 3 , is always the same wherever the point 2 may be; that is, the function $J\left(a_{2}\right)$ is independent of $a$. The point 2 may coincide with 1 , for the value $J\left(a_{1}\right)$ is the limit of $J\left(a_{2}\right)$ as $a_{2}$ approaches $a_{1}$. The value $t_{2}$ is the value of $t_{2}=t\left(a_{2}\right)$ defined by the equation $\left(E_{t a}\right)$ above.

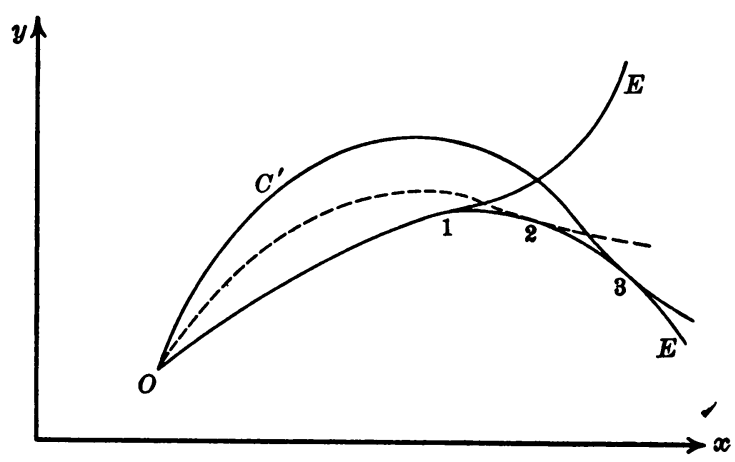

Fig. 5.

This theorem is proved in the following way. Differentiating $J\left(a_{2}\right)$ with respect to $a_{2}$ one obtains

$$
\begin{aligned}
\frac{d J\left(C_{02}\right)}{d a_{2}} & =\frac{d}{d a_{2}} \int_{t_{0}}^{t_{3}} F\left(\varphi, \psi, \varphi_{t}, \psi_{t}\right) d t \\
& =\left.F \frac{d t}{d a_{2}}\right|_{t_{0}} ^{t_{3}}+\int_{t_{0}}^{t_{3}}\left[F_{x} \varphi_{a}+F_{y} \psi_{a}+F_{x^{\prime}} \varphi_{a t}+F_{y^{\prime}} \psi_{a} t\right] d t .
\end{aligned}
$$

Now making the usual integration by parts of the last two members of the part under the integral sign and remembering that the curve under consideration is an extremal, and besides that $d t / d a_{2}, \varphi_{a}, \psi_{a}$ all vanish for $t=t_{0}$, one finally has

$$
\frac{d}{d a_{2}} J\left(C_{02}\right)=\left.F \frac{d t}{d a_{2}}\right|^{t_{2}}+\left.\left[F_{x^{\prime}} \varphi_{a}+F_{y^{\prime}} \psi_{a}\right]\right|^{t_{z}} .
$$

But since $F$ satisfies the homogeneity condition

this can be written

$$
x^{\prime} F_{x^{\prime}}+y^{\prime} F_{y^{\prime}}=F,
$$

$$
\frac{d}{d a_{2}} J\left(C_{02}\right)=\left.\left[F_{x^{\prime}}\left(\varphi_{t} \frac{d}{d a_{2}} t+\varphi_{a}\right)+F_{v^{\prime}}\left(\psi_{t} \frac{d}{d a_{2}} t+\psi_{a}\right)\right]\right|^{t_{2}},
$$

where the expressions in parentheses are exactly the derivatives $\bar{x}_{a}, \bar{y}_{a}$, as follows from the equations $\left(E_{x y}\right)$. Since at the point 2 the positive tangents to the 
extremal and envelope coincide it follows that

$$
\frac{d \varphi}{d t}=k \bar{x}_{a}, \quad \frac{d \psi}{d t}=k \bar{y}_{a} \quad(k>0) .
$$

And furthermore since the derivatives $F_{x^{\prime}}, F_{y^{\prime}}$ are positively homogenous with order zero, one has

$\frac{d}{d a_{2}^{-}} J\left(C_{02}\right)=\left.\left[F_{x^{\prime}}\left(x, y, x_{a}, y_{a}\right) x_{a}+F_{y^{\prime}}\left(x, y, x_{a}, y_{a}\right) y_{a}\right]\right|^{2}=\left.F\left(\bar{x}, \bar{y}, \bar{x}_{a}, \bar{y}_{a}\right)\right|^{2}$,

where $\bar{x}_{a}, \bar{y}_{a}$ are the direction cosines of the tangent to the envelope. But when the integral $J\left(E_{23}\right)$ along the envelope $E$ is considered and its derivative taken, one has

$$
\frac{d}{d a_{2}} J\left(E_{23}\right)=\frac{d}{d a_{2}} \int_{a_{2}}^{a_{l_{3}}} F\left(\bar{x}, \bar{y}, \bar{x}_{a}, \bar{y}_{a}\right) d a=-\left.F\left(\bar{x}, \bar{y}, \bar{x}_{a}, \bar{y}_{a}\right)\right|^{2} .
$$

Hence after adding, the sum of the derivatives turns out to have the value zero; i. e.,

$$
\frac{d}{d a_{2}} J\left(C_{02}\right)+\frac{d}{d a_{2}} J\left(E_{23}\right)=0,
$$

Therefore the sum of the integrals $J\left(C_{02}\right)$ and $J\left(E_{23}\right)$ is always the same, wherever the point 2 in Fig. 5 may be, and has the same value as that of the integral $J\left(C_{03}\right)$; i. e., its value where the point 2 coincides with 3 . Hence the value of the integral $J\left(a_{2}\right)$ is independent of $a . A$ similar statement holds for the other branch of the envelope.

§5. Determination of the locus of the points on the extremals at which the absolute minimum ceases.

The existence of more than one extremal through certain points of the field $R_{x y}$ having been shown for the V-shaped region near the cusp point $\left(x_{1}, y_{1}\right)$ it is now important to consider the relative va'ues furnished by these extremals through a given point of this region. The extremal arc through a point of the $V$-shaped region, corresponding to values $t(x, y), a(x, y)$ in the region $R_{t a}^{\prime \prime}$, can never furnish even a relative minimum in the field. For such an extremal is always tangent to the envelope before it intersects $(x, y)$, and the envelope theorem can be applied in the usual way to prove that such an arc cannot minimize.

It will be shown that starting from the point 1 a curve is uniquely determined which marks on each extremal of the fieid the point at which it ceases to provide an absolute minimum in comparison with other curves of the field.

Consider again the set of extremals $\left(C_{a}{ }^{\prime}\right)$ through the fixed point 0 , having 
the envelope $E$ with a cusp at the point 1 , as shown in Fig. 1. The extremal through the point 1 will have for equations

$$
x=\varphi\left(t, a_{1}\right), y=\psi\left(t, a_{1}\right) ;
$$

those extremals touching the envelope on the lower branch may be denoted by $\left(C^{\prime}\right)$.

$$
x=\varphi\left(t, a^{\prime}\right), \quad y=\psi\left(t, a^{\prime}\right), \quad a^{\prime}>a_{1} ;
$$

and the extremal touching the upper branch will have for equations

$$
x=\varphi(t, a), \quad y=\psi(t, a),
$$

Consider now an extremal $C^{\prime}$ cutting the envelope $E$ in a point 2 and tangent to it at a point 3 (see Fig. 1). Through every point 4 of the arc $C_{23}^{\prime}$ there passes one and but one extremal arc $C_{04}$ which contains no point of tangency with the envelope. For $C_{02}$ in particular it follows from the envelope law that one has the equality

$$
J\left(C_{02}\right)=J\left(C_{01}\right)+J\left(E_{12}\right),
$$

where 2 is the intersection of $C^{\prime}$ and the branch $a<a_{1}$ of $E$. But since Weierstrass' construction can be made along $C_{01}+E_{12}$ in the field of extremals $C^{\prime}$, it follows that

and hence

$$
J\left(C_{02}^{\prime}\right)<J\left(C_{01}\right)+J\left(E_{12}\right),
$$

Similarly

$$
J\left(C_{02}^{\prime}\right)<J\left(C_{02}\right)
$$

$$
J\left(C_{03}^{\prime}\right)>J\left(C_{03}\right),
$$

where 3 is the point of contact of $C^{\prime}$ with the branch $a>a_{1}$ of $E$. Consequently, from the continuity of $F$, it is seen that on the arc $C_{23}^{\prime}$ there is at least one point 4 between 2 and 3 such that one has the equality

$$
J\left(C_{04}^{\prime}\right)=J\left(C_{04}\right) \text {. }
$$

It will be shown that there is only one point 4 between 2 and 3 and one extremal $C_{04}$ for which equation (6) holds.

In as much as there is only one extremal of the set $C$ which passes through a given point of the region under consideration before it touches the envelope, it follows that any point on $C^{\prime}$ between the points 2 and 3 determines a unique extremal of this type which passes through it. In other words when $a^{\prime}$ is $\mathrm{g}$ ven, each $t^{\prime}$ between $t_{2}$ and $t_{3}$ determines one and but one set of values $(t, a)$ for which the equations

$$
\varphi\left(t^{\prime}, a^{\prime}\right)=\varphi(t, a), \quad \psi\left(t^{\prime}, a^{\prime}\right)=\psi(t, a)
$$

hold. Consequently both $t$ and $a$ may be considered as functions of $t^{\prime}$ and $a^{\prime}$ which are of class $C^{\prime}$ since $\Delta(t, a) \neq 0$, and after transposition of $J\left(C_{04}\right)$ the 
left member of equation (6) may be thought of as a function of $t^{\prime}$, say $W\left(t^{\prime}\right)$ when $a^{\prime}$ is fixed. Written out in full this function is

$$
\begin{aligned}
W\left(t^{\prime}\right)=\int_{t_{0}}^{t^{\prime}} F\left[\varphi\left(t^{\prime}, a^{\prime}\right),\right. & \left.\psi\left(t^{\prime}, a^{\prime}\right), \varphi_{t}\left(t^{\prime}, a^{\prime}\right), \psi_{t}\left(t^{\prime}, a^{\prime}\right)\right] d t^{\prime} \\
& -\int_{t_{0}}^{t} F\left[\varphi(t, a), \psi(t, a), \varphi_{t}(t, a), \psi_{t}(t, a)\right] d,
\end{aligned}
$$

where for simplicity the subscript 4 is omitted in the upper limits of the integrals. Hence the statement that there is only one point on $C_{23}^{\prime}$ for which equation (6) holds will be proved if it can be shown that there is only one value of $t^{\prime}$ in the range of values $t_{2}^{\prime} \leqq t^{\prime} \leqq t_{3}^{\prime}$ for which $W\left(t^{\prime}\right)$ vanishes. From (4) the values of $W\left(t_{2}^{\prime}\right)$ is negative, and on account of (5) it follows that $W\left(t_{3}^{\prime}\right)$ is positive. It will therefore be sufficient to show that the derivative of $W\left(t^{\prime}\right)$ has constantly the same sign in the given interval. On differentiating it is found that the derivative $W^{\prime}\left(t^{\prime}\right)$ becomes

$$
\begin{aligned}
W^{\prime}\left(t^{\prime}\right)=F\left[\varphi\left(t^{\prime}, a^{\prime}\right),\right. & \left.\psi\left(t^{\prime}, a^{\prime}\right), \varphi_{t}\left(t^{\prime}, a^{\prime}\right), \psi_{t}\left(t^{\prime}, a^{\prime}\right)\right]\left.\right|^{4} \\
& -\frac{\partial a}{\partial t^{\prime}} \int_{t_{0}}^{t} \frac{\partial}{\partial a} F\left[\varphi(t, a), \psi(t, a), \varphi_{t}(t, a), \psi(t, a)\right] d t \\
& -\left.\frac{\partial t}{\partial t^{\prime}} F\left[\varphi(t, a), \psi(t, a), \varphi_{t}(t, a), \psi_{t}(t, a)\right]\right|^{4},
\end{aligned}
$$

which for brevity will be written in the form

$$
W^{\prime}\left(t^{\prime}\right)=\left.F\left(t^{\prime}, a^{\prime}\right)\right|^{4}-\left.F(t, a) \frac{\partial t}{\partial t^{\prime}}\right|^{4}-\frac{\partial a}{\partial t^{\prime}} \int_{t_{0}}^{t} \frac{\partial}{\partial a} F(t, a) d t .
$$

Now consider the last term of this derivative. On differentiating out it becomes

$$
\int_{t_{0}}^{t} \frac{\partial}{\partial a} F(t, a) d t=\int_{t_{0}}^{t}\left[F_{x} \varphi_{a}+F_{y} \psi_{a}+F_{x^{\prime}} \varphi_{t a}+F_{y^{\prime}} \psi_{t a}\right] d t .
$$

After making the usual integration by parts and remembering that the curves considered are extremals all of which pass through the point 0 , we obtain the following result:

$$
\int_{t_{0}}^{t} \frac{\partial}{\partial a} F(t, a) d t=F_{x^{\prime}}(t, a) \varphi_{a}(t, a)+\left.F_{y^{\prime}}(t, a) \psi_{a}(t, a)\right|^{4} .
$$

Hence the derivative becomes

$$
\begin{aligned}
W^{\prime}\left(t^{\prime}\right)=F\left(t^{\prime}, a^{\prime}\right)- & F(t, a) \frac{\partial t}{\partial t^{\prime}} \\
& -F_{x^{\prime}}(t, a) \varphi_{a}(t, a) \frac{\partial a}{\partial t^{\prime}}-\left.F_{y^{\prime}}(t, a) \psi_{a}(t, a) \frac{\partial a}{\partial t^{\prime}}\right|^{4} .
\end{aligned}
$$


In order to simplify this expression consider the derivatives of the equations of condition (7) at the point 4 . It is readily verified that the following relations hold:

$$
\begin{aligned}
& \varphi_{a}(t, a) \frac{\partial a}{\partial t^{\prime}}=\varphi_{t^{\prime}}\left(t^{\prime}, a^{\prime}\right)-\varphi_{t}(t, a) \frac{\partial t}{\partial t^{\prime}}, \\
& \psi_{a}(t, a) \frac{\partial a}{\partial t^{\prime}}=\psi_{t^{\prime}}\left(t^{\prime}, a^{\prime}\right)-\psi_{t}(t, a) \frac{\partial t}{\partial t^{\prime}} .
\end{aligned}
$$

Substituting these values we have equation (8) in the form

$$
\begin{aligned}
W^{\prime}\left(t^{\prime}\right)=F\left(t^{\prime}, a^{\prime}\right) & -F_{x^{\prime}}(t, a) \varphi_{t^{\prime}}\left(t^{\prime}, a^{\prime}\right)-F_{y^{\prime}}(t, a) \psi_{t^{\prime}}\left(t^{\prime}, a^{\prime}\right) \\
& -\left.\frac{\partial t}{\partial t^{\prime}}\left[F(t, a)-F_{x^{\prime}}(t, a) \varphi_{t}(t, a)-F_{y^{\prime}}(t, a) \psi_{t}(t, a)\right]\right|^{4} .
\end{aligned}
$$

On account of the homogeneity condition which the function $F$ satisfies it is seen that the coefficient of $\partial t / \partial t^{\prime}$, vanishes. Hence written out in full the derivative of $W\left(t^{\prime}\right)$ is

$$
\begin{aligned}
W^{\prime}\left(t^{\prime}\right)= & F\left[\varphi\left(t^{\prime}, a^{\prime}\right), \psi\left(t^{\prime}, a^{\prime}\right), \varphi_{t}\left(t^{\prime}, a^{\prime}\right), \psi_{t}\left(t^{\prime}, a^{\prime}\right)\right] \\
& -F_{x^{\prime}}\left[\varphi(t, a), \psi(t, a), \varphi_{t}(t, a), \psi_{t}(t, a)\right] \varphi_{t}\left(t^{\prime}, a^{\prime}\right) \\
& -\left.F_{v^{\prime}}\left[\varphi(t, a), \psi(t, a), \varphi_{t}(t, a), \psi_{t}(t, a)\right] \psi_{t}\left(t^{\prime}, a^{\prime}\right)\right|^{t},
\end{aligned}
$$

that is

$$
W^{\prime}\left(t^{\prime}\right)=\left.E\left[\varphi(t, a), \psi(t, a), \varphi_{t}(t, a), \psi_{t}(t, a), \varphi_{t}\left(t^{\prime}, a^{\prime}\right), \psi_{\iota}\left(t^{\prime}, a^{\prime}\right)\right]\right|^{4},
$$

where $E$ is the well-known Weierstrass function.* From this it follows, since $F_{1}>0$, that the derivative is always positive; and therefore the function $W\left(t^{\prime}\right)$ is monotonically increasing as $t^{\prime}$ takes all values in the interval $t_{2}^{\prime} \leqq t^{\prime} \leqq t_{3}^{\prime}$. But inasmuch as $W\left(t^{\prime}\right)$ is negative at the point 2 and positive at 3 it follows that there is but one value of $t^{\prime}$ for which $W\left(t^{\prime}\right)$ vanishes and therefore but one point 4 on the extremal $C^{\prime}$ for which equation (6) holds.

Hence by means of equations (7), which express the fact that $t$ and $a$ are functions of $t^{\prime}$ and $a^{\prime}$, the equation

$$
\begin{aligned}
& W\left(t^{\prime}, a^{\prime}\right)=\int_{t_{0}}^{t} F\left[\varphi\left(t^{\prime}, a^{\prime}\right), \psi\left(t^{\prime}, a^{\prime}\right), \varphi_{t}\left(t^{\prime}, a^{\prime}\right), \psi_{t}\left(t^{\prime}, a^{\prime}\right)\right] d^{t} \\
&-\int_{t_{0}}^{t} F\left[\varphi(t, a), \psi(t, a), \varphi_{t}(t, a), \psi_{t}(t, a)\right] d t
\end{aligned}
$$

may be regarded as defining $t^{\prime}$ as a function, $t^{\prime}=T\left(a^{\prime}\right)$, of $a^{\prime}$. Moreover this is a single valued function of class $C^{\prime}$ for $t_{2}^{\prime} \leqq t^{\prime}=t_{3}^{\prime}$, since by the above for

* See Bolza, loc. cit., $\$ 306$. 
every $a^{\prime}$ there is only one value of $t^{\prime}$ for which the equation holds, and the derivative $\partial I V / \partial t^{\prime}$ is different from zero. A continuous curve $M$ is thus defined by means of the equations

$$
x=\varphi\left[T\left(a^{\prime}\right), a^{\prime}\right], \quad y=\psi\left[T\left(a^{\prime}\right), a^{\prime}\right],
$$

wh ch has the property that it passes through the point 1 , as ${ }^{\circ} \mathrm{s}$ seen at once when it is noticed that for $a^{\prime}=a$, the equation $W\left(t^{\prime}, a^{\prime}\right)=C$ is satisfied by the values $t^{\prime}=t_{1}, t=t_{1}$, and $a=a_{1}$. Consequently it follows that there exists a curve $M$ of class $C^{\prime}$ in the $V$-shaped region of the field (see Fig. 1 ), which passes through the point 1 and has the property that any extremal arc $C_{04}^{\prime}$ which does not intersect $M$ gives a smaller value to the integral $J$ than the value given by the corresponding extremal $C_{04}$. On the other hand if $C_{04}^{\prime}$ intersects $M$ then the integral value $J\left(C_{04}^{\prime}\right)$ is greater than $J\left(C_{04}\right)$.

As a corollary to these results it follows that as the point 4 moves from 0 along the extremal $C^{\prime}$, the arc $C_{04}^{\prime}$ ceases to furnish an absolute minimum for the integral $J$ in the field when the point 4 coincides with the intersection of $C^{\prime}$ and $M$.

Similar statements hold for the extremals of the set $C$.

\section{§. Sufficient conditions for an absolute minimum in the field.}

With the aid of the curve $M$ determined in the preceding section it will be possible to show that an extremal arc $C_{02}$ which does not contain the point of intersection of $C$ with the curve $M$ furnishes a smaller value to the integral than any other admissible curve lying entirely in the field. A similar statement holds for the extremals $C^{\prime}$. In the following the proof will be given for the set $C$. The proof for the second case can be made in an entirely analogous manner.

It will be necessary to compare $C_{02}$ with three kinds of variations: (1) when the variation $V_{02}$ joins 0 with the extremity 2 of $C_{02}$ in the leaf $L_{x y}^{\prime}$, and lies entirely within the leaf, i. e., the continuous set of values $(t, a)$ a'ong $V_{02}$ all belong to the leaf $L_{x y}^{\prime}$; (2) when $V_{02}^{\prime}$ lies entirely within the leaf but joins 0 to the point of the leaf underneath 2 ; (3) when $V_{02}^{\prime \prime}$ crosses the boundary of the leaf. These cases are illustrated in Fig. 4.

Consider first the case when the curve $V$ lies entirely in the leaf $L_{x y}^{\prime}$. From the usual theory of the calculus of variations it is known that the extremal $C_{02}$ furnishes a smaller value to the integral than any such admissible curve. For along the curve $V$ the Weierstrass construction is possible, and by hypothesis the $E$-function is always positive. In case 2 ) it is known for the same reason that

$$
J\left(V_{02}^{\prime}\right)>J\left(C_{02}^{\prime}\right),
$$

and furthermore on account of the position of 2 with respect to the curve $M$ 
on the $\operatorname{arc} C_{02}$, that

$$
J\left(C_{02}^{\prime}\right)>J\left(C_{02}\right) .
$$

Hence in either of the two cases the extremal $C_{02}$ furnishes a smaller value to the integral than any admissible curve of the types denoted by 1) and 2) which lie in the field.

It remains now to consider the case when the curve $V^{\prime \prime}$ crosses the boundary of $L_{x y}^{\prime}$, i. e., when at some point of $V_{02}^{\prime \prime}$ the corresponding values of $(t, a)$ belong to the envelope $E$. It will be necessary to consider only the case when $V^{\prime \prime}$ crosses the boundary once, for if it should have any finite number of crossings the methods of proof would be the same. In Fig. 4 let $V_{02}^{\prime \prime}$ be such a curve. Denote its point of intersection with the boundary by 3 . Then from the envelope law and the usual theory it results that

$$
J\left(C_{01}\right)+J\left(E_{13}\right)=J\left(C_{03}\right)<J\left(V_{03}^{\prime \prime}\right) .
$$

But the curve $C_{01}+E_{13}+V_{32}^{\prime \prime}$ is an admissible curve in the field of extremals, of type $\mathrm{V}$, and therefore the Weierstrass construction holds. In consequence of this, one has the inequality

$$
J\left(C_{02}\right)<J\left(C_{01}\right)+J\left(E_{13}\right)+J\left(V_{32}^{\prime \prime}\right),
$$

and therefore as a result of the preceding

$$
J\left(C_{02}\right)<J\left(V_{02}^{\prime \prime}\right)+J\left(V_{32}^{\prime \prime}\right)=J\left(V_{02}\right) .
$$

Hence an admissible curve of the kind considered in case (3) also does not furnish as small a value to the integral as the extremal arc $C_{02}^{\prime}$. Therefore the extremal arc $C_{02}^{\prime}$ actually furnishes a smaller value to the integral $J$ than any other admissible curve in the field, provided that it does not contain the intersection point of $C^{\prime}$ and $M$.

The University of Chicago. 